\title{
«Die universitäre Förderung der Hausarzt- medizin entspricht einem zentralen Bedürfnis»
}

\author{
Er ist der erste - und vorerst einzige - Professor für Hausarztmedizin in der \\ Schweiz: Peter Tschudi, praktizierender Hausarzt und Co-Leiter des Instituts \\ für Hausarztmedizin an der Universität Basel (IHAMB). Seine Ernennung zum \\ Titularprofessor wurde vom Basler Universitätsrat am 27. April dieses Jahres \\ genehmigt. Die SÄZ befragte Peter Tschudi sowie den Dekan der Medizini- \\ schen Fakultät der Universität Basel, Prof. André Perruchoud, zur Bedeutung \\ dieses Schritts für die Hausarztmedizin in der Schweiz.
}

SÄZ: Dass ein Hausarzt sich habilitiert und Professor für Hausarztmedizin wird, notabene an der ältesten Universität der Schweiz, ist für helvetische Verhältnisse ein bisher singuläres Ereignis und sicher ein Höhepunkt einer individuellen Medizinerkarriere. War der Weg zur Professur in Ihrem Fall Bestandteil einer langfristigen Karriereplanung, oder hat sich das «einfach so» ergeben?

Peter Tschudi: Die Beförderung zum Titularprofessor ist für mich Ausdruck der Wertschätzung der Universität Basel und der Medizinischen Fakultät für das rund 25jährige intensive Engagement der Hausärzte in unserer Region im Bereich der Lehre und Forschung. Bereits im April 2003 bekannte sich die Fakultätsversammlung an der Universität Basel zur Hausarztmedizin als profilbildendem Fach in der Lehre und erkannte, dass die Förderung der Hausarztmedizin universitär und gesundheitspolitisch einem zentralen Bedürfnis entspricht. Persönlich habe ich diesen Karriereschritt nie geplant, bin ich doch im

«Ich hoffe, dass dieser Präzedenzfall an den übrigen Universitäten Schule machen wird»

Grunde vergleichsweise «alt» dafür. Die Habilitation war nicht nur für mich persönlich eine interessante Herausforderung; sie war auch gewissermassen die erfolgreiche Nagelprobe dafür, dass die Hausarztmedizin definitiv in den universitären Strukturen unseres Landes als den eta- blierten klinischen und präklinischen Disziplinen gleichgestelltes Fach anerkannt wird. Mein eigentliches Ziel ist und bleibt die Förderung und Etablierung der Hausarztmedizin auf universitärer Ebene.

Die Bedeutung, die Ihre Ernennung zum Titularprofessor für die Hausarztmedizin hat, ist sicher nicht zu unterschätzen. Ein Indiz dafür ist auch die mediale Resonanz.

Auf dem langen Weg der Hausarztmedizin in Basel ist meine Professur ein «kleiner normaler» Zwischenschritt: 1979 Gründung der Basler Arbeitsgruppe Ausbildung der SGAM, 1984 erste offizielle fakultäre Kommission für Hausarztmedizin, 1994 Forum für Interdisziplinäre Hausarztmedizin FIHAM Basel, 2005 Institut für Hausarztmedizin Basel IHAMB, 2007 erste Habilitation und Professur in Hausarztmedizin - und 2009 strukturelles Ordinariat für Hausarztmedizin an der Medizinischen Fakultät Basel? Nun hoffe ich, dass dieser Präzedenzfall der Professur in Basel an den übrigen Universitäten Schule machen wird.

Die bereits bestehenden oder sich anbahnenden Probleme der medizinischen Grundversorgung in der Schweiz - Stichworte: Probleme der Tarifstruktur, Organisation des Notfalldienstes, Nachwuchsmangel - werden seit einiger Zeit in den Medien breit thematisiert. Was bedeutet Ihre Ernennung für die Sache der Hausarztmedizin in der Schweiz?

Mit unseren Forschungsprojekten in der Gesundheitsversorgung können wir fundierte Daten sammeln und damit einen wesentlichen Beitrag leisten zur Planung der Zukunft der Hausarztme- 


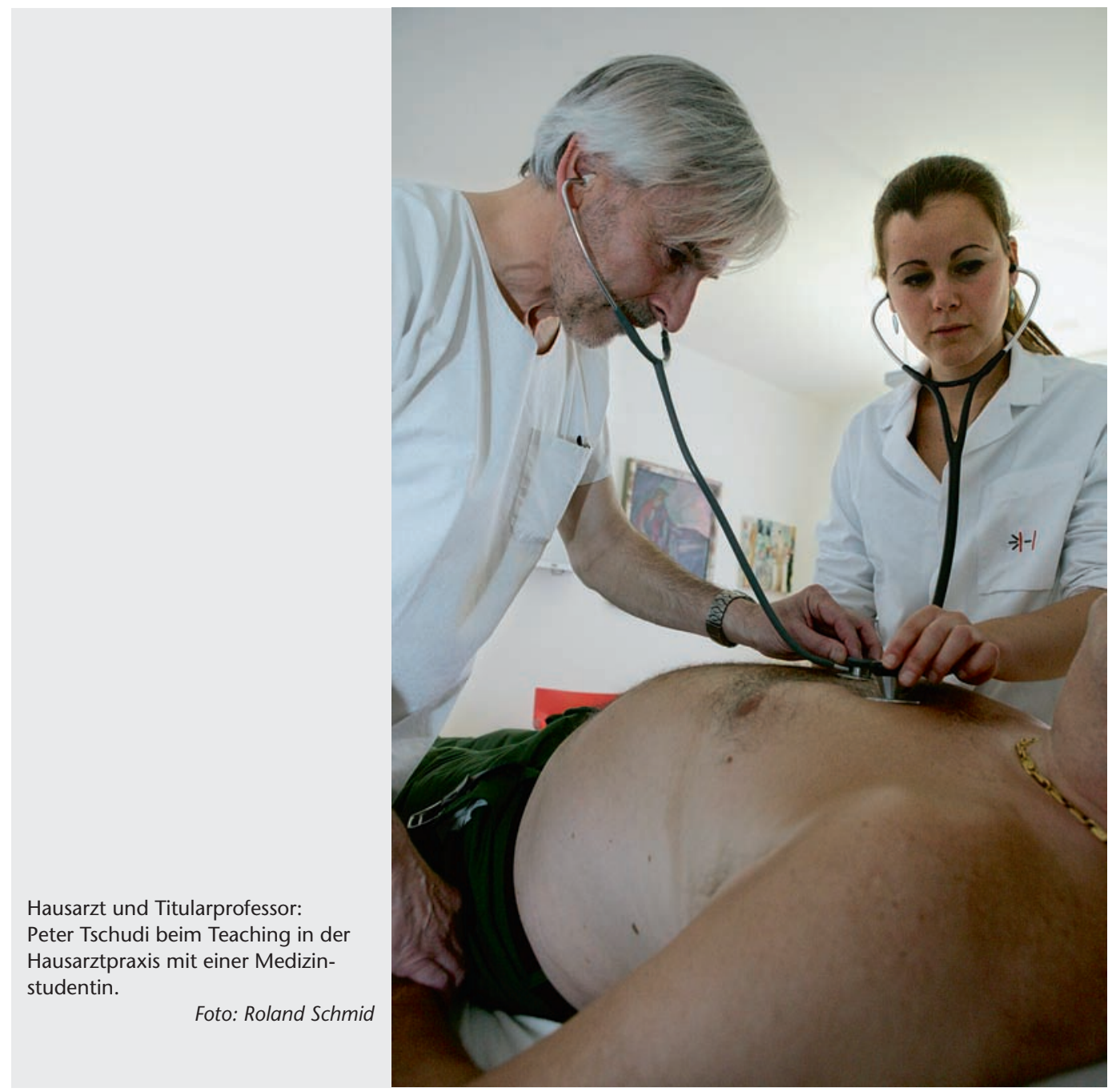

dizin und des Berufsbildes Hausarzt. In diesem Zusammenhang kann etwa die vom BAG unterstützte Berufszielstudie genannt werden, in deren Rahmen seit 2002 jährlich die Medizinstudierenden in Basel befragt werden. Ein weiteres Beispiel ist die Workforce-Studie, die die Erhebung der Praxistätigkeit 2005 der Hausärzte Schweiz und 2006 der Hausärzte Basel-Stadt und Basel-Landschaft umfasst. Zudem engagieren wir uns auch für Projekte über neue Notfalldienstmodelle. Wir suchen die Kooperation mit der Spitalleitung des Universitätsspitals im Hinblick auf eine bedarfsgerechte und optimierte Versorgung der Notfallpatienten durch Spital- und Hausärzte, zum Beispiel durch eine der Notfallstation vorgeschaltete Hausarztpraxis, die für die Lehre durch Einbindung von Studierenden, als Weiterbildungsstelle für angehende Hausärzte und für Forschungsprojekte prädestiniert wäre.

Das erste und schweizweit bis jetzt immer noch einzige Institut für Hausarztmedizin an der Universität
Basel, das Sie als Co-Leiter führen, besteht seit 2005. Wenn Sie nach den ersten zwei Jahren für diese Institution Bilanz ziehen: Was wurde erreicht bzw. nicht erreicht, wie sehen die Perspektiven aus?

Zusammen mit meinem Co-Leiter Prof. Benedict Martina sind wir aktuell ein Leitungsteam von acht Hausärztinnen und Hausärzten, die zum Teil über Drittmittel finanziert sind: Dr. Klaus Bally, Dr. Hans-Ruedi Banderet, Dr. Ursina Halter, Dr. Simon Heiniger, Dr. Ruedi Isler, Dr. Silvana Romerio-Bläuer. Auf eine Kurzformel gebracht, kann die Entwicklung als insgesamt sehr erfreulich bezeichnet werden. Geht man etwas mehr ins Detail, lassen sich verschiedene Punkte hervorheben. So ist unsere Vernetzung in der Region Nordwestschweiz mit den Hausärzten, Spezialisten und Spitälern ausgezeichnet. Unsere Lehre, vor allem das Einzeltutoriat, erhält von den Studierenden im Rating Bestnoten. Die Wertschätzung durch die Medizinische Fakultät für unsere Lehre hat letztlich auch dazu geführt, dass wir ein derart umfassendes Pensum in allen Jahres- 
kursen anbieten können und für die Mitarbeit in entscheidenden fakultären Kommissionen angefragt wurden. Unsere Forschung in Hausarztmedizin, die wir neu aufgebaut haben, ist gut gestartet. Wir haben grosse innovative Forschungsprojekte wie die Hypertoniedatenbank Hc.cH (www.hypertensioncohort.ch) entwickelt und gestartet. Dies lässt sich unter anderem daran messen, dass in den nächsten Tagen sieben Dissertanten ihre Arbeiten aus dem Forschungsbereich der Hausarztmedizin einreichen werden, die unter unserer Leitung entstanden sind. Gerade weil sich die universitäre Hausarztmedizin erfreulich entwickelt und an Bedeutung gewinnt - nicht nur in Basel! -, benötigen wir mehr personelle und finanzielle Ressourcen.

Wie erleben Sie die Zusammenarbeit mit und die Akzeptanz durch Ihre Kollegen an der Medizinischen Fakultät? Sind Sie als Allgemeinmediziner gleichwertiger Kollege, oder spüren Sie - unausgesprochene «innerfakultäre Standesunterschiede»? Gibt es offene oder versteckte Widerstände gegen die "Akademisierung der Hausarztmedizin»?

Das Klima der Zusammenarbeit mit Vertretern der Medizinischen Fakultät, mit Vertretern anderer Fakultäten, mit den Spitalärzten und mit niedergelassenen Haus- und Spezialärzten erachte ich als ausgezeichnet. Dies ist nicht zuletzt dadurch bedingt, dass unsere Lehrtätigkeit an der Universität seit Jahren auf Kooperation basiert und in einem Klima der Offenheit und gegenseitigen Akzeptanz sowie Wertschätzung stattgefunden hat. Wenn es von uns Hausärzten als bereichernd empfunden wird, in gewissen Lehr-, Fortbildungs- und Forschungsgebieten auf die wertvolle Hilfe der Spezialisten inner- und ausserhalb der Universität zählen zu dürfen, so gilt dies auch umgekehrt. Unsere Fakultätskollegen schätzen es durchaus, wenn die Lehre, Fortbildung und Forschung in ihrem Fachgebiet durch Beiträge von Hausärzten unterstützt wird. Noch verbessert werden könnte die Förderung der angehenden Hausärztinnen und Hausärzte in der Weiterbildung, indem geeignete Assistentenstellen angeboten würden. Doch auch in diesem Punkt laufen Gespräche, und wir sind auf gutem Weg.

Wie sind Sie mit den Standes- und Dachorganisationen der Hausärzte - Schweizerische Gesellschaft für Allgemeinmedizin SGAM, Kollegium für Hausarztmedizin KHM - vernetzt? Inwiefern können diese Organisationen von Ihrer universitären Arbeit profitieren?
Die Vernetzung über die Mitarbeit in diversen Arbeitsgruppen und Kongressen mit der SGAM und dem KHM ist gut. Ich wünschte mir des öfteren eine pointiertere Stellungnahme und klarere Reaktionen unserer gewerkschaftlichen Standesorganisationen, da dies für die Nachwuchsförderung, für die Profilbildung und das Berufsbild des zukünftigen Hausarztes wichtig, ja entscheidend ist. Mit meinem Engagement kämpfe ich für die Stärkung der Hausarztmedizin, für ein verbessertes Image und Arbeitsbedingungen - namentlich Teilzeitarbeit, Gruppenpraxis, Notfalldienst usw. -, für mehr Kompetenzen und Verantwortung beim Hausarzt und für eine bessere bzw. adäquate Entlöhnung. Mit

\section{«Unsere Lehre, vor allem das Einzeltutoriat, erhält von den Studierenden im Rating}

\section{Bestnoten»}

unseren Forschungsprojekten (z.B. WorkforceStudie Schweiz und Basel-Stadt/Basel-Landschaft) können wir unsere Standesorganisationen mit fundiertem eigenem Zahlenmaterial beliefern, das sie in den Gesprächen und Verhandlungen mit den politischen Behörden und Versicherern verwenden können.

Zu welchen Themen der Hausarztmedizin besteht für Sie vordringlich Forschungsbedarf? Wo setzen Sie Ihre persönlichen Schwerpunkte?

Ein mir wichtiges Anliegen ist, die klinische patientenorientierte Forschung in der Hausarztpraxis aufzubauen. Doch dafür sind wir auf die Unterstützung und Förderung durch den Nationalfonds und andere entsprechende Gremien angewiesen - nicht mehr nur mit Worten, sondern mit Taten! Das Institut für Hausarztmedizin Basel IHAMB hat verschiedene Forschungsschwerpunkte in den Bereichen Ausbildung, Qualitätssicherung und Epidemiologie, Gesundheitspolitik sowie qualitative Forschung gesetzt, über die sich Interessierte im Detail auf unserer Homepage unter www.ihamb.unibas.ch informieren können. Ein wichtiges Projekt ist zudem die Gründung und der Aufbau eines Hausarztforschungsnetzwerks Nordwestschweiz. Wir in Basel freuen uns auf diese Forschungstätigkeit und laden alle Hausärztinnen und Hausärzte ein, sich an unseren Forschungsprojekten vom Hausarzt für den Hausarzt zu beteiligen. 